Homology, Homotopy and Applications, vol.5(1), 2003, pp.601-612

\title{
ON THE DERIVATIVE OF THE STABLE HOMOTOPY OF MAPPING SPACES
}

\author{
JOHN R. KLEIN \\ (communicated by Gunnar Carlsson)
}

Abstract

Using the chain rule, we give a homotopy theoretic approach to identifying the derivative of the functor $X \mapsto Q_{+}\left(X^{K}\right)$.

\section{Introduction}

1.1.

The calculus of homotopy functors is a method invented by Goodwillie to organize information about homotopy functors from spaces to spaces (see $[\mathbf{G o}]$ ). Central to the theory is the notion of differentiating a homotopy functor $f$ at a based space $X$. Roughly, the derivative $\partial f(X)$ is defined to be the spectrum whose $j$-th space is the homotopy fiber of the map $f\left(X \vee S^{j}\right) \rightarrow f(X)$ (cf. 2.6 below). Under mild assumptions, the derivative measures the homotopical behavior of the functor in a certain stable range.

The goal of the present work is to describe an alternative approach to computing the derivative of the functor

$$
X \quad \mapsto Q_{+} X^{K} .
$$

Here $K$ denotes a finite complex, $X^{K}$ denotes the space of unbased maps $K \rightarrow X$ and $Q_{+}$denotes unreduced stable homotopy. When $K$ is the circle, this functor arises in Waldhausen's algebraic $K$-theory of spaces (see e.g., [B-C-C\&]).

The derivative of $Q_{+} X^{K}$ was first determined by Goodwillie using framed bordism theory in [Go]. Another approach using configuration spaces can be found in $[\mathbf{H e}]$ and independently in $[\mathbf{A r}]$. Both of these approaches rely on manifold theory (the configuration space approach uses the fact that $K$ has the homotopy type of a parallelizable manifold with boundary). The approach of this paper is manifold free.

\section{2 .}

The description of Goodwillie's formula for the derivative of $Q_{+} X^{K}$ will require some preparation. Let $E \rightarrow K$ be a (Hurewicz) fibration. For each $i \geqslant 0$, let $E_{i}$ denote the pushout of the diagram

$$
K \leftarrow S^{i-1} \times E \stackrel{\subset}{\rightarrow} D^{i} \times E .
$$

Received August 2, 2002, revised November 18, 2003; published on December 31, 2003.

2000 Mathematics Subject Classification: Primary: 55P65, Secondary: 55P91, 18G55, 55P42.

Key words and phrases: Homotopy functor, spectrum, mapping space.

(C) 2003, John R. Klein. Permission to copy for private use granted. 
The induced map $E_{i} \rightarrow K$ is then another fibration equipped with a preferred section. Its fiber obtained from the original fiber by adding a disjoint basepoint and $i$-fold suspending. Let $\gamma^{E_{i}}(K)$ denote the (based) space of all sections of $E_{i} \rightarrow K$. As $i$ varies, these spaces form a spectrum (after a minor rectification; see [Go, 0.1]). Let $\gamma^{S_{+}{ }^{E}}(K)$ denote the corresponding $\Omega$-spectrum.

We apply the preceding in a special case. Let $x \in X$ be a choice of basepoint. Define a fibration

$$
E_{x}(K, X) \rightarrow K
$$

with total space

$$
E_{x}(K, X):=\{(k, f) \mid k \in K, f: K \rightarrow X, f(k)=x\}
$$

(topologized as a subspace of $K \times X^{K}$ ) and map to $K$ given by the first coordinate projection. Note that the fiber over $k \in K$ is the function space of maps $K \rightarrow X$ sending $k$ to $x$. Using the above construction, we obtain a spectrum

$$
\gamma^{S_{+}^{\bullet} E_{x}(K, X)}(K) \text {. }
$$

We are now in a position to state Goodwillie's result [Go, 2.4].

Theorem 1.3 (Goodwillie). Let $(X, x)$ be a based space, $K$ a finite $C W$ complex, and assume that $X^{K}$ is connected. Then the derivative of the functor $X \mapsto Q_{+} X^{K}$ at $(X, x)$ is given by the spectrum $\gamma^{S_{+}^{\bullet} E_{x}(K, X)}(K)$.

(note: if $X$ is $r$-connected and $\operatorname{dim} K \leqslant r$, then $X^{K}$ is connected.)

\section{4 .}

Our approach to 1.3 will use the chain rule of $[\mathbf{K}-\mathbf{R}]$. For a composite functor $g \circ f$ satisfying suitable technical hypotheses, the chain rule says that its derivative has the homotopy type of the homotopy orbit spectrum

$$
\partial g(f(X)) \wedge_{h \Omega f(X)} \partial f(X)
$$

for a certain naturally defined action of the loop group $\Omega f(X)$ on both $\partial f(X)$ and $\partial g(f(X))$.

The chain rule gives a different description of the derivative of $Q_{+} X^{K}$. Using elementary manipulations with homotopy limits, we will show that the chain rule description is homotopy equivalent to Goodwillie's description (this will require replacing $K$ within its homotopy type by a finite simplicial complex). Our proof is manifold and configuration space free.

The chain rule step was already provided in $[\mathbf{K}-\mathbf{R}]$. The idea is that the functor in question can be written as a composite consisting of the mapping space functor $X \mapsto X^{K}$ followed by the unreduced stable homotopy functor $Y \mapsto Q_{+} Y$.

To describe the chain rule result, let $G=\Omega_{x} X$ denote the realization of the Kan loop group of the total singular complex of $X$ (this is a topological group model of 
the loop space of $X$ based at $x$. Let $S^{0}[G]:=S^{0} \wedge G_{+}$be the suspension spectrum of $G \amalg \mathrm{pt}$.

Let $F\left(K, S^{0}[G]\right)$ denote the function spectrum of maps from $K$ into $S^{0}[G]$. Explicitly, this is the spectrum whose $j$-th space is the function space $F\left(K, Q\left(S^{j} \wedge G_{+}\right)\right)$. Give the function spectrum an action of the topological group $G^{K}$ via pointwise left multiplication, i.e., by

$$
\phi * \lambda \quad:=\quad(k \mapsto \phi(k) \cdot \lambda(k)),
$$

for $\phi \in G^{K}$ and $\lambda \in F\left(K, Q\left(S^{j} \wedge G_{+}\right)\right)$, where $G$ acts on $Q\left(S^{j} \wedge G_{+}\right)$by left translation on $G_{+}$(and trivially on the suspension coordinates). Then $F\left(K, S^{0}[G]\right.$ ) is a spectrum with naive $G^{K}$-action.

We now state the outcome of the chain rule computation.

Proposition 1.5 (Ex. 12.4 of $[\mathbf{K}-\mathbf{R}])$. Let $(X, x)$ be a based space. Let $K$ be a finite $C W$ complex such that $X^{K}$ is connected. Then the derivative of $X \mapsto Q_{+} X^{K}$ at $(X, x)$ is given by the homotopy orbit spectrum

$$
F\left(K, S^{0}[G]\right)_{h G^{K}}
$$

where $G=\Omega_{x} X$.

Goodwillie's formula is a direct consequence of 1.5 together with the following theorem, which is the main result of this paper:

Theorem 1.6. Let $X$ be a space and let $K$ be a finite simplicial complex such that $X^{K}$ is connected. Then there is a weak equivalence of spectra

$$
\gamma^{S_{+}^{\bullet} E_{x}(K, X)}(K) \simeq F\left(K, S^{0}[G]\right)_{h G^{K}} .
$$

\subsection{Outline}

$\S 2$ is preliminary material. In $\S 3$ we interpret the homotopy limits of certain kinds of functors as section spaces. The proof of Theorem 1.6 is contained in $\S 4$. The proof of Lemma 3.4 is the content of $\S 5$.

\subsection{Acknowledgments}

This paper constitutes a major revision of a Bielefeld SFB343 preprint bearing the same title dating from August, 1995. Much of the research for this project was done while I was a guest of the Norwegian Academy of Sciences in Oslo, Norway in June, 1995. I wish to thank Bjørn Jahren and John Rognes for their hospitality, for their interest, and for engaging discussions. I am indebted to Bill Dwyer for sketching me a proof of Lemma 3.4.

This paper is the outcome of an attempt to understand why the chain rule for the derivative of $X \mapsto Q_{+} X^{K}$ yields an expression which is prima facie different from Goodwillie's formula. A guiding principle for this project was distilled by Bill Browder: "Homotopy theoretic statements should have homotopy theoretic proofs."

\footnotetext{
${ }^{1}$ Note Added in Proof: Goodwillie has recently discovered another manifold free derivation of the formula for the derivative of $Q_{+} X^{K}$ (see [Go]).
} 


\section{Preliminaries}

\subsection{Spaces}

In this paper Top denotes the category of compactly generated spaces. In particular, we make the convention that products are to be re-topologized using the compactly generated topology. Function spaces are topologized using the compact open topology. If $Z$ is a space, then $Z_{+}$denotes the based space given by taking the disjoint union of $Z$ with a single point.

A weak equivalence of spaces is shorthand for (a chain of) weak homotopy equivalence(s). A weak equivalence is denoted by $\stackrel{\sim}{\rightarrow}$, whereas, we often write chains of weak equivalences using $\simeq$ (the same notation will be used when discussing weak equivalences of spectra).

\subsection{Spectra}

A spectrum $X$ is a collection of based spaces $\left\{X_{i}\right\}_{i \in \mathbb{N}}$ together with based maps $\Sigma X_{i} \rightarrow X_{i+1}$ where $\Sigma X_{i}$ denotes the reduced suspension of $X_{i} . X$ is an $\Omega$-spectrum if each of the adjoint maps $X_{i} \rightarrow \Omega X_{i+1}$ are weak equivalences. The sphere spectrum $S^{0}$ is the spectrum whose $j$-space is $S^{j}$ and whose structure maps are the identity.

A map of spectra $X \rightarrow Y$ consists of maps $X_{i} \rightarrow Y_{i}$ which are compatible with the structure maps. A weak equivalence $X \rightarrow Y$ is a map which induces an isomorphism on homotopy groups. Every spectrum $X$ comes equipped with a natural weak equivalence $X \stackrel{\sim}{\rightarrow} X^{\prime}$ where $X^{\prime}$ is an $\Omega$-spectrum. The category of spectra is denoted by $\mathbf{S p}$.

If $Y$ is a based space, and $E$ is a spectrum, then $E \wedge Y$ is the spectrum whose $j$-th space is the smash product $E_{j} \wedge Y$. This will have the correct homotopy type whenever the spaces of $E$ and $Y$ have the homotopy type of CW complexes.

If $Z$ is an unbased space $F(Z, E)$ will denote the function spectrum whose $j$-th space is $\left(E_{j}\right)^{Z}$. The function spectrum has the correct homotopy type if $Z$ has the homotopy type of a $\mathrm{CW}$ complex and $E$ is an $\Omega$-spectrum. Henceforth, by slight abuse of notation, we make the following convention: if $E$ isn't an $\Omega$-spectrum, then $F(K, E)$ is defined to be $F\left(K, E^{\prime}\right)$ where $E^{\prime}$ is the $\Omega$-spectrum associated with $E$.

\section{3. $\quad G$-spectra}

Let $G$ be a topological group object of Top. For technical reasons, we require that the underlying space of $G$ is a CW complex. A (naive) $G$-spectrum is a spectrum $X$ such that each $X_{i}$ is a based (left) $G$-space and each structure map $\Sigma X_{i} \rightarrow X_{i+1}$ is equivariant, where the action of $G$ on $\Sigma X_{i}$ is defined so as to act trivially on the suspension coordinate.

Maps of $G$-spectra are maps of spectra that are compatible with the $G$-action. A weak equivalence of $G$-spectra is a map which is a weak equivalence of underlying unequivariant spectra. S. Schwede has shown that this notion of weak equivalence arises from a Quillen model category structure on $G$-spectra (see [Sc]). In this model structure, a fibrant object is a $G$-spectrum $X$ which is an $\Omega$-spectrum: A cofibrant object is (the retract of) a $G$-spectrum $X$ such that $X_{n}$ is built up from a point by attaching free $G$-cells (i.e., $D^{n} \times G$ ), moreover, the structure maps $\Sigma X_{n} \rightarrow X_{n+1}$ are given by attaching free $G$-cells to $\Sigma X_{n}$. 


\subsection{Homotopy orbits}

If $X$ is a $G$-spectrum such that the underlying spaces $X_{j}$ have the homotopy type of $\mathrm{CW}$ complex, then the homotopy orbit spectrum $X_{h G}$ is the (non-equivariant) spectrum whose $j$-space is the orbits of $G$ acting diagonally on " $X_{j}$ made free":

$$
X_{j} \wedge_{G} E G_{+} .
$$

Here $E G$ denotes the free contractible $G$-space, arising from the bar construction (if $X$ is an arbitrary $G$-spectrum, we can always functorially replace it within it's weak homotopy type by a $G$-spectrum whose spaces are CW complexes). If $X$ happens to be cofibrant, then $X_{h G}$ is weak equivalent to the orbit spectrum $X / G$. If $G$ acts trivially on $X$, the homotopy orbit spectrum is just $X \wedge B G_{+}$.

\subsection{Homotopy limits}

The basic reference on homotopy limits is $[\mathbf{B}-\mathbf{K}]$ (see also $[\mathbf{D}-\mathbf{S}]$ for another approach). We give the definition of holim in the special case of finite poset shaped diagrams of spaces (the construction in the case of spectra is analogous, except that spectra should be replaced with $\Omega$-spectra prior performing the construction). We will follow $[\mathbf{B}-\mathbf{K}]$, except that we work contravariantly and with spaces rather than with simplicial sets.

Let $f: D \rightarrow$ Top be a contravariant functor, where $D$ is a finite simplicial complex considered as a poset by inclusion of simplices. The limit of $f$ is the space of natural transformations

$$
\operatorname{Map}(*, f)
$$

where $*$ denotes the constant functor with value a point. The limit is to be topologized as a subspace of $\prod_{s \in D} f(s)$.

The homotopy limit of $f$ is the space of natural transformations

$$
\operatorname{Map}(|D /-|, f)
$$

where, for each $s \in D$, the space $|D / s|$ is the space consisting of simplices $t$ such that $s$ is a face of $t$ (i.e., the star of $s$ ). Topologize the homotopy limit as a subspace of the product $\prod_{s} f(s)^{|D / s|}$.

The unique natural transformation from $|K /-| \rightarrow *$ gives a map

$$
\lim f \rightarrow \operatorname{holim} f .
$$

A natural transformation of $f \rightarrow g$ functors is said to be an objectwise weak equivalence if $f(d) \rightarrow g(d)$ is a (weak) equivalence for every $d \in D$. More generally, we say that $f$ and $g$ are objectwise weak equivalent, if there exists a finite chain of objectwise weak equivalences that connect $f$ to $g$. The following is well known (for a proof see [B-K, XI $\S 6])$ :

Lemma 2.6. If $f \rightarrow g$ is an objectwise weak equivalence, then the induced map

$$
\operatorname{holim} f \rightarrow \operatorname{holim} g
$$

is a weak equivalence. 
Notation. For a contravariant functor $f$ we denote the homotopy limit in one of three ways: as holim $f$, as $\operatorname{holim}_{D} f$ or as $\operatorname{holim}_{s \in D} f(s)$.

\subsection{Definition of the derivative}

We outline the construction of the derivative of a homotopy functor, omitting many details (for a more complete account, see $[\mathbf{G o}]$ and $[\mathbf{K}-\mathbf{R}]$ ). Although not used in the proof of the main result, this material is included for the purpose of making the paper more self-contained.

Let

$$
f: \text { Top } \rightarrow \text { Top }_{*}
$$

be a homotopy functor from spaces to based spaces. If $X$ is a based space and $j \geqslant 0$ is an integer, define

$$
\partial_{j} f(X):=\operatorname{fiber}\left(f\left(X \vee S^{j}\right) \rightarrow f(X)\right)
$$

(where "fiber" denotes homotopy fiber).

We next briefly indicate the definition of structure maps

$$
\Sigma \partial_{j} f(X) \rightarrow \partial_{j+1} f(X)
$$

making the collection $\left\{\partial_{j} f(X)\right\}_{j} \geqslant 0$ into a spectrum, denoted $\partial f(X)$ and called the derivative of $f$ at $X$.

By considering $S^{j+1}$ as the union of its hemispheres, we have a pushout diagram

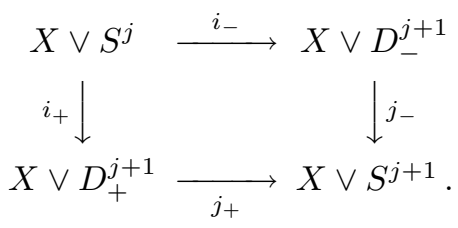

Applying $f$ to this diagram, we obtain

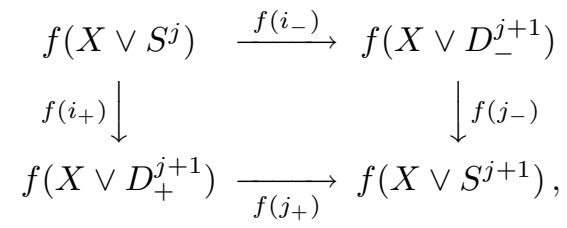

and hence a chain of maps

$$
\partial_{j} f(X) \simeq \operatorname{fiber}\left(f\left(i_{+}\right)\right) \rightarrow \operatorname{fiber}\left(f\left(j_{-}\right)\right) \simeq \Omega \partial_{j+1} f(X) .
$$

Hence taking adjoints, we get a weak map $\Sigma \partial_{j} f(X) \rightarrow \partial_{j+1} f(X)$. Goodwillie then shows how to rectify the above to give an actual spectrum.

The derivative has additional structure: using the homotopy fiber sequence

$$
\partial_{j} f(X) \rightarrow f\left(X \vee S^{j}\right) \rightarrow f(X)
$$

one can equip the fiber $\partial_{j} f(X)$ with an action of a topological group model for the loop space $\Omega f(X)$. This gives $\partial f(X)$ the structure of an $\Omega f(X)$-spectrum. 


\section{Homotopy limits as section spaces}

\subsection{Mapping spaces}

Let $K$ be a finite geometric simplicial complex. We will abuse notation slightly and identify simplices $s$ of $K$ with their corresponding geometric simplices $\Delta^{\operatorname{dim} s}$.

Let $\Delta_{K}$ be the category whose objects are simplices of $K$ and whose morphisms are the inclusions of such simplices.

Let $X$ be a space. Define a contravariant functor

$$
\Delta_{K} \rightarrow \text { Top }
$$

by the rule $s \mapsto X^{s}$.

Lemma 3.2. The evident map

$$
X^{K}=\lim _{s \in \Delta_{K}} X^{s} \rightarrow \operatorname{holim}_{s \in \Delta_{K}} X^{s}
$$

is a weak equivalence.

Proof. This is a special case of [Dw, Prop. 3.8].

\subsection{Section spaces}

We generalize the above to section spaces of fibrations. If $p: E \rightarrow K$ is a fibration and $L \subset K$ is a subcomplex, define

$$
\gamma^{E}(L):=\text { Space of sections of } p \text { along } L \text {. }
$$

Applying $\gamma^{E}$ to simplices defines a contravariant functor

$$
\gamma^{E}: \Delta_{K} \rightarrow \text { Top }
$$

Lemma 3.4. The evident map

$$
\gamma^{E}(K)=\lim \gamma^{E} \rightarrow \operatorname{holim} \gamma^{E}
$$

is a weak equivalence.

The proof is deferred to $\S 5$.

\subsection{Spectrification}

If $L \subset K$ is a subcomplex, then we can form the spectrum $\gamma^{S_{+}^{\bullet} E_{L}}(L)$ as in the introduction, where $E_{L} \rightarrow L$ is the restriction of $p: E \rightarrow K$ along $L$. Specializing to simplices, we obtain a contravariant functor

$$
\gamma^{S_{+} E}: \Delta_{K} \rightarrow \mathbf{S p} .
$$

Corollary 3.6. The evident map

$$
\gamma^{S_{+}^{\bullet} E}(K) \rightarrow \operatorname{holim} \gamma^{S_{+}^{\bullet} E}
$$

is a weak equivalence.

Proof. Apply 3.4 to each fibration $E_{i} \rightarrow K$ and then assemble. 
3.7.

For $s \in \Delta_{K}$, define a space

$$
F_{x}(K / s, X):=\{f: K \rightarrow X \mid f(s)=x\}
$$

(i.e., the space of based maps $K / s \rightarrow X$ ). The assignment

$$
s \quad \mapsto \quad S^{0} \wedge F_{x}(K / s, X)_{+}
$$

is then a contravariant functor $\Delta_{K} \rightarrow \mathbf{S p}$ which we denote by $S^{0} \wedge F_{x}(K /-, X)_{+}$.

The next proposition recasts Goodwillie's expression for the derivative of $Q_{+} X^{K}$ in terms suitable for manipulating as a homotopy limit.

Proposition 3.8. There is a weak equivalence of spectra

$$
\gamma^{S_{+}^{\bullet} E_{x}(K, X)}(K) \simeq \operatorname{holim}_{s \in \Delta_{K}} S^{0} \wedge F_{x}(K / s, X)_{+} \cdot
$$

Proof. By 2.6 and 3.6, it is enough to construct an objectwise weak equivalence

$$
S^{0} \wedge F_{x}(K /-, X)_{+} \stackrel{\sim}{\rightarrow} \gamma^{S_{+}^{\bullet} E_{x}(K, X)} .
$$

We first exhibit a natural transformation

$$
\phi: F_{x}(K /-, X) \rightarrow \gamma^{E_{x}(K, X)} .
$$

If $s$ is a simplex of $K$, then a point of $F_{x}(K / s, X)$ consists of a map $f: K \rightarrow X$ with $f(s)=x$. To specify point of $\gamma^{E_{x}(K, X)}(s)$, it is sufficient to define a map $g: s \times K \rightarrow X$ with the property that $g(t, t)=x$ for all $t \in s$. Let $\phi(f): s \times K \rightarrow X$ be the map given by $\phi(f)(t, k)=f(k)$. Then $\phi(f)$ is a point of $\gamma^{E_{x}(K, X)}(s)$. It is straightforward to check that $\phi$ defines a natural transformation.

We next assert that $\phi$ is an objectwise weak equivalence. For each $s \in \Delta_{K}, \phi(s)$ may be described as a map of based function spaces

$$
F_{x}(K / s, X) \rightarrow F_{x}((s \times K) / s, X)
$$

where $s \subset s \times K$ is the diagonal inclusion. This map is induced by the second factor projection

$$
(s \times K) / s \rightarrow K / s
$$

and the latter is clearly a homotopy equivalence. Consequently, $\phi(s)$ is a weak equivalence.

If $E_{x}(K, X)_{j} \rightarrow K$ is the fibration of the introduction given by unreduced fiberwise suspension of the fibers of $E_{x}(K, X) \rightarrow K$, the forgoing generalizes to give an objectwise weak equivalence

$$
\phi_{j}: S_{+}^{j} \wedge F_{x}(K /-, X) \stackrel{\sim}{\rightarrow} \gamma^{E_{x}(K, X)_{j}}
$$

(we omit the details). The $\phi_{j}$, taken together describe the desired weak equivalence of spectra.

\section{Proof of Theorem 1.6}

4.1.

The proof will be based on two lemmas. 
Lemma 4.2. The functor $Y \mapsto Y_{h G}$ from $G$-spectra to spectra commutes with finite homotopy limits up to weak equivalence.

Proof. The homotopy orbit construction preserves homotopy cocartesian squares. In the category of $G$-spectra, homotopy cocartesian squares are the same thing as homotopy cartesian squares. The result now follows by observing that any finite homotopy limit can be written as a finite iterated homotopy pullback.

For the second lemma, suppose that $G \rightarrow Q$ is a surjective homomorphism (arising from a surjective map of simplicial groups). Let $H$ denote its kernel.

Lemma 4.3. Let $Y$ be a $G$-spectrum. Then there is a natural weak equivalence of spectra

$$
Y_{h G} \simeq\left(Y_{h H}\right)_{h Q} .
$$

Proof. We can assume that $Y$ is a cofibrant $G$-spectrum. Then $Y$ is also a cofibrant $H$-spectrum. Let $Y_{G}$ denote the orbit spectrum $Y / G$. Then

$$
\begin{aligned}
Y_{h G} & \simeq Y_{G} \quad \text { since } Y \text { is } G \text {-cofibrant, } \\
& =\left(Y_{H}\right)_{Q} \\
& \simeq\left(Y_{H}\right)_{h Q} \quad \text { since } Y_{H} \text { is } Q \text {-cofibrant } \\
& \simeq\left(Y_{h H}\right)_{h Q} \quad \text { since } Y \text { is } H \text {-cofibrant. }
\end{aligned}
$$

Here, the passage from the second to the third line makes use of observation that $Y \wedge_{H} E G_{+}$is a cofibrant $Q$-spectrum. The passage from the third to the fourth line uses the fact that $E G$ is a model for $E H$.

\section{4 .}

We now commence with the proof of Theorem 1.6. By 3.2 generalized to spectra, the evident map

$$
F\left(K, S^{0}[G]\right)=\lim _{s \in \Delta_{K}} F\left(s, S^{0}[G]\right) \rightarrow \operatorname{holim}_{s \in \Delta_{K}} F\left(s, S^{0}[G]\right)
$$

is a weak equivalence of $G$-spectra. In fact, this map is $G^{K}$-equivariant, provided that we let $G^{K}$ act on $F\left(s, S^{0}[G]\right)$ via the restriction homomorphism $G^{K} \rightarrow G^{s}$.

Since $\Delta_{K}$ is finite, and homotopy orbits are homotopy invariant, we infer from 4.2:

Lemma 4.5. The evident map of spectra

$$
F\left(K, S^{0}[G]\right)_{h G^{K}} \rightarrow \operatorname{holim}_{s \in \Delta_{K}} F\left(s, S^{0}[G]\right)_{h G^{K}} .
$$

is a weak equivalence.

In view of 4.5 and 3.8 , the proof of Theorem 1.6 is complete once we show:

Proposition 4.6. There is an objectwise weak equivalence

$$
F\left(-, S^{0}[G]\right)_{h G^{K}} \simeq S^{0} \wedge F_{x}(K /-, X)_{+}
$$


Proof. For $s \in \Delta_{K}$, consider the (exact) sequence of topological groups

$$
F_{*}(K / s, G) \longrightarrow G^{K} \stackrel{\text { restriction }}{\longrightarrow} G^{s} \text {. }
$$

Then $F_{*}(K / s, G)$ is the kernel of the restriction homomorphism and by 4.3 we obtain an objectwise weak equivalence of functors

$$
F\left(s, S^{0}[G]\right)_{h G^{K}} \simeq\left(F\left(s, S^{0}[G]\right)_{h F_{*}(K / s, G)}\right)_{h G^{s}} .
$$

By definition of action of $G^{K}$ on $F\left(s, S^{0}[G]\right)$, we find that this action restricts to the trivial action on the subgroup $F_{*}(K / s, G)$. We therefore have an objectwise weak equivalence

$$
\left.F\left(s, S^{0}[G]\right)_{h F_{*}(K / s, G)}\right)_{h G^{s}} \simeq\left(F\left(s, S^{0}[G]\right) \wedge F_{x}(K / s, X)_{+}\right)_{h G^{s}},
$$

where we identify $B F_{*}(K / s, G)$ with $F_{x}(K / s, X)$ (this identification is valid because $F_{x}(K / s, X)$ is connected $)$.

Next observe that the homomorphism $G \rightarrow G^{s}$, given by mapping a group element $g$ to the constant function with value $g$, is a $G$-equivariant homotopy equivalence. We infer from this that there is an objectwise weak equivalence

$$
\left(F\left(s, S^{0}[G]\right) \wedge F_{x}(K / s, X)_{+}\right)_{h G^{s}} \simeq\left(F\left(s, S^{0}[G]\right) \wedge F_{x}(K / s, X)_{+}\right)_{h G} .
$$

Finally, observe that the map of $G$-spectra

$$
S^{0}[G] \rightarrow F\left(s, S^{0}[G]\right)
$$

which sends a point to the constant function at that point is a an equivariant weak equivalence. Substituting, we obtain objectwise weak equivalences

$$
\begin{aligned}
\left(F\left(s, S^{0}[G]\right) \wedge F_{x}(K / s, X)_{+}\right)_{h G} & \simeq\left(S^{0}[G] \wedge F_{x}(K / s, X)_{+}\right)_{h G} \\
& \simeq S^{0} \wedge F_{x}(K / s, X)_{+} .
\end{aligned}
$$

Assembling the above information, we get an objectwise weak equivalence of functors

$$
F\left(s, S^{0}[G]\right)_{h F(K, G)} \simeq S^{0} \wedge F_{x}(K / s, X)_{+},
$$

as was to be proved.

\section{Proof of Lemma 3.4}

Recall that $\gamma^{E}: \Delta_{K} \rightarrow$ Top is the contravariant functor defined by

$$
\gamma^{E}(s)=\text { space of sections of } E \rightarrow K \text { along } s .
$$

We wish to prove that that map $\lim \gamma^{E} \rightarrow \operatorname{holim} \gamma^{E}$ is a weak equivalence. The argument we give is due to Bill Dwyer.

\section{Step 1:}

Fact: the category of contravariant functors

$$
\Delta_{K} \rightarrow \text { Top }
$$

comes equipped with the structure of a model category in which 
- the weak equivalences are the natural transformations which are objectwise weak equivalences,

- the cofibrations are the natural transformations which are objectwise cofibrations, and

- the fibrations are described as follows: given a contravariant functor $f: \Delta_{K} \rightarrow$ Top and an object $s \in \Delta_{K}^{\mathrm{op}}$, define the matching space $M(f, s)$ by

$$
M(f, s)=\lim _{t \in \partial s} f(t)
$$

where $\partial s$ is the poset of simplices which are faces of $s$.

There is a natural map

$$
f(s) \rightarrow M(f, s) .
$$

A natural transformation $f \rightarrow g$ is then defined to be a fibration if (and only if) for each object $s \in \Delta_{K}$, the natural map

$$
F(s) \rightarrow g(s) \times_{M(g, s)} M(f, s)
$$

is a fibration.

For a proof this gives a model structure, see $[\mathbf{H o}, \S 5.2]$ or $[\mathbf{H i}]$. See also $[\mathbf{D}-\mathbf{S}, \S 10]$.

\section{Step 2:}

We claim that the contravariant functor

$$
\gamma^{E}: \Delta_{K} \rightarrow \text { Top }
$$

is fibrant with respect to this model category structure. This amounts to checking that the map

$$
\gamma^{E}(s) \rightarrow \gamma^{E}(\partial s)
$$

is a fibration for each $s \in \Delta_{K}$ (here, $\partial s$ denotes the geometric realization of the poset of faces of $s$ ). But this is a special case of a known property of section spaces of fibrations.

\section{Step 3:}

Recall that holim $\gamma_{E}$ is the space of natural maps

$$
\operatorname{Map}\left(\left|\left(\Delta_{K}\right) /-\right|, \gamma^{E}\right) .
$$

Observe that the natural transformation

$$
\left|\left(\Delta_{K}\right) /-\right| \rightarrow *
$$

is a weak equivalence between cofibrant objects of the category of contravariant functors. Since $\gamma^{E}$ is fibrant, this weak equivalence between cofibrant objects induces a weak equivalence of natural mapping spaces

$$
\lim \gamma^{E}=\operatorname{Map}\left(*, \gamma^{E}\right) \stackrel{\sim}{\rightarrow} \operatorname{Map}\left(\left|\left(\Delta_{K}\right) /-\right|, \gamma^{E}\right)=\operatorname{holim} \gamma^{E} .
$$




\section{References}

[Ar] Arone, G.: A generalization of the Snaith-type filtration. Trans. Amer. Math. Soc. 351, 1123-1150 (1999)

[B-C-C\&] Bökstedt, M., Carlsson, G., Cohen, R., Goodwillie, T., Hsiang, W., Madsen, I.: On the algebraic $K$-theory of simply connected spaces. Duke Math. J. 84, 541-563 (1996)

[B-K] Bousfield, A. K., Kan, D. M.: Homotopy Limits, Completions and Localizations. (LNM, Vol. 304). Springer 1972

[D-S] Dwyer, W., Spaliński, J.: Homotopy theories and model categories. In: Handbook of algebraic topology, pp. 73-126. North-Holland 1995

[Dw] Dwyer, W.: The centralizer decomposition of BG. In: Proceedings 1994 Barcelona Conference, Progress in Math. 136 , pp. 16-184. Birkhäuser 1996

[Go] Goodwillie, T. G.: Calculus I, the derivative of a homotopy functor. K-theory 4, 1-27 (1990)

[Go] Goodwillie, T. G.: Calculus III: Taylor series. Geometry and Topology 7, 645-711 (2003)

[He] Hesselholt, L.: A homotopy theoretic derivation of $Q \operatorname{MAP}(K, X)_{+}$. Math. Scan. 70, 193-203 (1992)

[Hi] Hirschhorn, P.: Model categories and their localizations. (Mathematical Surveys and Monographs, Vol. 99). Amer. Math. Soc. 2003

[Ho] Hovey, M.: Model categories. (Mathematical Surveys and Monographs, Vol. 63). Amer. Math. Soc. 1993

[K-R] Klein, J., Rognes, J.: A chain rule in the calculus of homotopy functors. Geometry and Topology 6, 853-887 (2002)

[Sc] Schwede, S.: Spectra in model categories and applications to the algebraic cotangent complex. J. Pure Appl. Algebra 120, 77-104 (1997)

This article may be accessed via WWW at http://www.rmi.acnet.ge/hha/ or by anonymous ftp at

ftp://ftp.rmi.acnet.ge/pub/hha/volumes/2003/n1a21/v5n1a21.(dvi,ps,pdf)

John R. Klein klein@math.wayne.edu

Department of Mathematics, Wayne State University,

Detroit, MI 48202,

USA 